\title{
Research on the modality and critical speed in the long axis of the rotor system
}

\author{
Na Zhao ${ }^{1, a}$, Juan XU ${ }^{1, b}$ and Haiyan Bie ${ }^{2}$ \\ ${ }^{1}$ Shandong Provincial Key Laboratory of Ocean Environment Monitoring Technology, Institute of \\ Oceanographic Instrumentation, Shandong Academy of Sciences, Zhejiang Road 28, Qingdao \\ 266001, China \\ ${ }^{2}$ College of Chemistry and Chemical Engineering, Ocean University of China \\ azhaona_sdioi@163.com, bujuan101066@163.com
}

Keywords: rotor system; finite element method; modal analysis; critical speed

\begin{abstract}
The finite-element linear analysis model in the typical rotor system is established by finite element method to analyze the modality and critical speed. The result shows that modes of vibration change greatly with different modalities in the rotor system. Torsion angle of the rotor increases gradually along the rotor axis and it gradually tends to a stable state near the turntable. Three order critical speeds in rotor bearing system are $190 \mathrm{rad} / \mathrm{s}, 442 \mathrm{rad} / \mathrm{s}$ and 890rad/s. With the support from rigid support to elastic support, the critical speed greatly reduces.
\end{abstract}

\section{Introduction}

In the vibrating machine with variable load such as long distance vibrating conveyer and probability vibrating screen, the loads change greatly and most are shock load and alternate load, which short the life of the vibration motor as power source and vibration source. At the same time the multi-diameter plastoelastic deformation will accelerate the vibration motor failure [1-4]. Therefore doing the harmonic analysis to rationally design the size and structure is one of the main to solve premature failure in such situations. The finite element analysis has a very wide application in engineering. And the finite element model and mesh generation are the main factors in effecting the analysis speed and accuracy. Reasonable modeling and mesh generation can make the analysis fast and accurate. While improper modeling and mesh generation will slow the solving speed, increase the error and even stop the solution. So combined with practical work, the vibration and critical speed analysis are done based on the finite element method in this paper.

\section{Finite element calculation and computational model}

The 3D model of the rotor system is made up of the rotor, other rigid bodies, low end coupling, gearbox and high end coupling. And when making model, the order is followed. Flexible part is included in the model and small time step is set to ensure the computer accuracy, which may increase the computing time greatly and reduce efficiency. So in the calculation the model in close to the real condition is simplified. In the vibrating system, without considering the mass of the transmission axis, the prime mover, driving gear, driven gear and load are set as four moment of inertia elements. Therefore the model is 4 of freedom torsional vibration system. The freedoms are the vibration displacements $\theta_{m}, \theta_{p}, \theta_{g}$ and $\theta_{L}$. So the system - analysis models are follows.

$$
\begin{aligned}
& I_{m} \ddot{\theta}_{m}+c_{p}\left(\dot{\theta}_{m}-\dot{\theta}_{p}\right)+k_{p}\left(\theta_{m}-\theta_{p}\right)=T_{m} \\
& I_{p} \ddot{\theta}_{p}+c_{p}\left(\dot{\theta}_{p}-\dot{\theta}_{m}\right)+k_{p}\left(\theta_{p}-\theta_{m}\right)+R_{p} W_{d}=0 \\
& I_{g} \ddot{\theta}_{g}+c_{g}\left(\dot{\theta}_{g}-\dot{\theta}_{L}\right)+k_{g}\left(\theta_{g}-\theta_{L}\right)-R_{g} W_{d}=0 \\
& I_{L} \ddot{\theta}_{L}+c_{p}\left(\dot{\theta}_{L}-\dot{\theta}_{g}\right)+k_{g}\left(\theta_{L}-\theta_{g}\right)=-T_{L}
\end{aligned}
$$


$\theta_{m}, \theta_{p}, \theta_{g}$ and $\theta_{L}$ are four moment of inertia elements. $C_{L}$ and ${ }^{C_{g}}$ are torsional damping of the driving and driven coupling shaft. $k_{p}$ and $k_{g}$ are torsion stiffness of the driving and driven coupling shaft. $T_{m}$ and $T_{L}$ are torsion acting on the prime mover and the load. ${ }^{W}$ is the dynamic meshing force of the gear.

Dynamic meshing force of the gear $W_{d}$ is

$W_{d}=c_{m}\left(R_{p} \dot{\theta}_{p}-R_{g} \dot{\theta}_{g}-\dot{e}\right)+k_{m}\left(R_{p} \theta_{p}-R_{g} \theta_{g}-e\right)$

The torsional vibration analysis model of the gear-rotor system is obtained. The matrix form is as follows.

$$
\begin{aligned}
& {[m]\{\ddot{\delta}\}+[c]\{\dot{\delta}\}+[c]\{\delta\}=\{P\}} \\
& {[\delta]=\left\{\theta_{m} \theta_{p} \theta_{R} \theta_{L}\right\}^{T}} \\
& {[m]=\left[\begin{array}{cccc}
I_{m} & & & \\
& I_{p} & & 0 \\
& & I_{g} & \\
& 0 & & I_{L}
\end{array}\right]} \\
& {[m]=\left[\begin{array}{cccl}
c_{p} & -c_{p} & 0 & 0 \\
-c_{p} & c_{p}+R_{p}^{2} c_{m} & -c_{m} R_{p} R_{g} & 0 \\
0 & -c_{m} R_{p} R_{g} & c_{g}+R_{g}^{2} c_{m} & -c_{g} \\
0 & 0 & -c_{g} & c_{g}
\end{array}\right]} \\
& {[k]=\left[\begin{array}{cccl}
k_{p} & -k_{p} & 0 & 0 \\
-k_{p} & k_{p}+R_{p}^{2} k_{m} & -k_{m} R_{p} R_{g} & 0 \\
0 & -k_{m} R_{p} R_{g} & k_{g}+R_{g}^{2} k_{m} & -k_{g} \\
0 & 0 & -k_{g} & k_{g}
\end{array}\right]} \\
& {[p]=\left[\begin{array}{l}
T_{m} \\
-c_{m} R_{g} \dot{e}-R_{p} k_{m} e \\
c_{m} R_{g} \dot{e}+R_{g} k_{m} e \\
-T_{L}
\end{array}\right]}
\end{aligned}
$$

$\{\delta\}$ is the vbration displacement array. $[m],[c]$ and $[k]$ are mass matrix, damping matrix and stiffness matrices. $\{P\}$ is the load array.

The rotor system is simplified in computing. The rotor is simplified as the rigid disc and revolving axis is simplified as the elastic axis with a distributed mass. The revolving axis and the rotor are described as the axis element and the disk element. The axis element is taken as the elastic element, and there are the left and right nodes in each axis element. The disk element is taken as the inertial element with a lumped mass, append to one node of the axis element. 3D model of the rotor is shown in Fig.1. The vibration of the rotor is the key point in this paper. So the grid is divided and modal calculation is done to obtain the response results of the torsional vibration. 


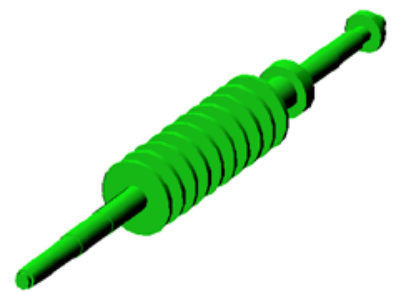

Fig 1 Finite element model of the rotor system

\section{Rotor dynamic analysis}

The modes of vibration of all orders are showed in Fig.2. The mode of vibration is max in the first-order modal. While in the second-order modal the mode shows the S-shape change. In third-order modal, there is a hill - shaped model of vibration. And the change occurs slowly in the forth-order modal. A diagram of the rotor in different locations, with angular changes is shown in Fig.3. Along the rotor axis, torsion angle increases greatly. And it gradually tends to a stable state near the turntable.

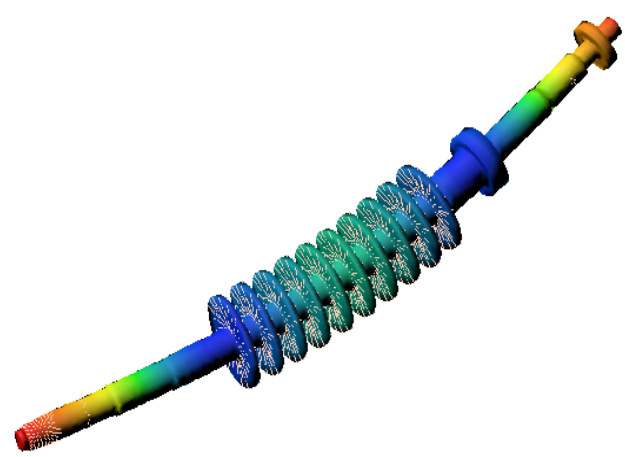

(a) the first-order modal

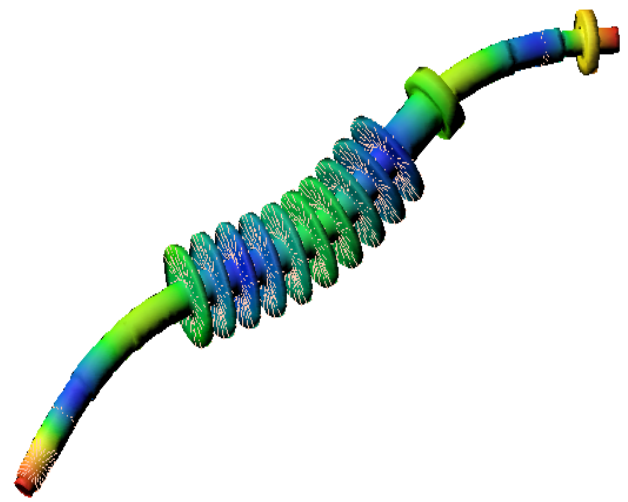

(c) the third-order modal

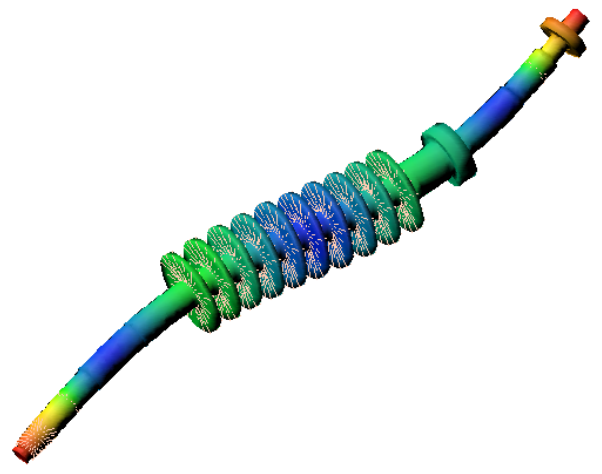

(b) the second-order modal

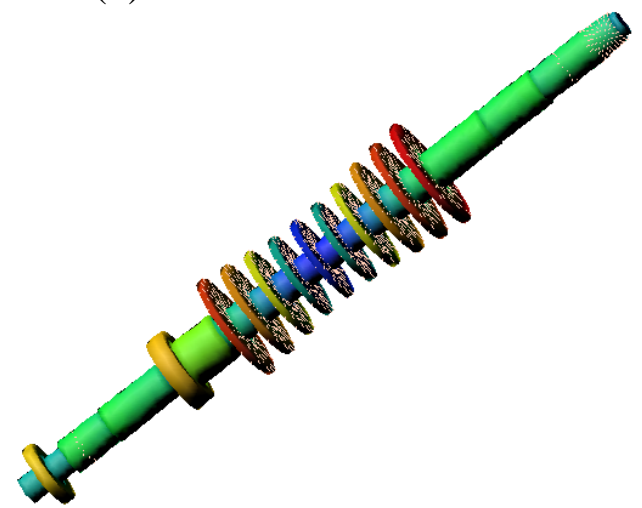

(d) the forth-order modal

Fig 2 The modes of vibration of all orders

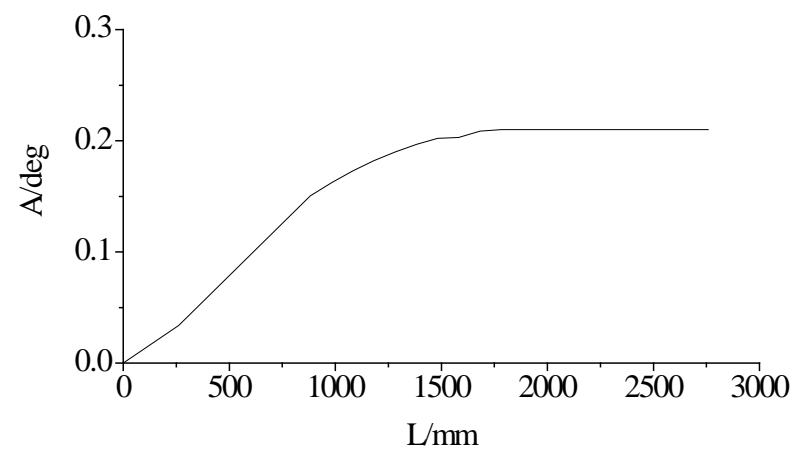

Fig 3 The diagram of the rotor in different locations, with angular changes 
Traditionally amplitude for synchronous forward whirl is only considered in calculation the critical speed. That is because in the rotor operation the synchronous forward whirling dominates the rotor vibration, owing to the unbalanced force. The critical speed generally refers to the speed with synchronous forward whirl. From the Campbell chart 4, three order critical speeds in rotor bearing system are $190 \mathrm{rad} / \mathrm{s}, 442 \mathrm{rad} / \mathrm{s}$ and 890rad/s. Therefore with the support from rigid support to elastic support, the critical speed greatly reduces.

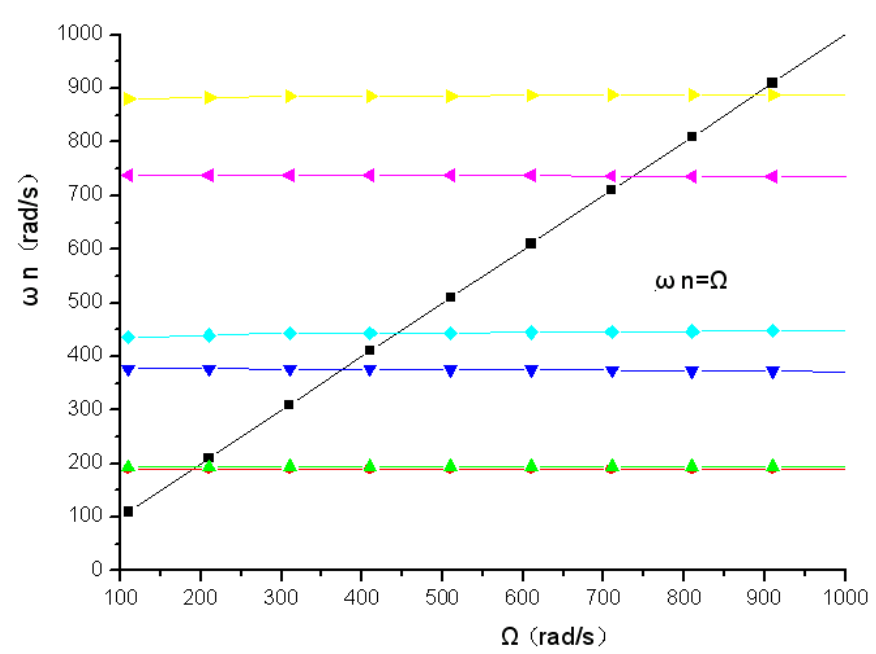

Fig 4 Campbell chart of the rotor

\section{Conclusions}

The finite-element linear analysis model in the typical rotor system is established by finite element method to analyze the modality and critical speed. The result shows that modes of vibration change greatly with different modalities in the rotor system. Torsion angle of the rotor increases gradually along the rotor axis and it gradually tends to a stable state near the turntable. Three order critical speeds in rotor bearing system are $190 \mathrm{rad} / \mathrm{s}, 442 \mathrm{rad} / \mathrm{s}$ and 890rad/s. With the support from rigid support to elastic support, the critical speed greatly reduces.

\section{Acknowledgements}

This work was financially supported by the scientific research foundation of Shandong province Outstanding Young Scientist Award (No: BS2013NJ017) and Qingdao science and technology program of basic research projects (No: 13-1-4-210-jch and 13-1-4-248-jch).

\section{References}

[1] Luo, W., Accurate FEM/BEM simulation of wireless passive surface acoustic wave sensors. High-Performance Ceramics V, Pts 1 and 2, 2008. 368-372: p. 198-201.

[2] S. Merz, et al., Development of coupled FE/BE models to investigate the structural and acoustic responses of a submerged vessel. Journal of Computational Acoustics. Vol. 15(2007), p. 23-47.

[3] Lee, D.H., Optimal placement of constrained-layer damping for reduction of interior noise. Aiaa Journal, Vol. 46(2008), p. 75-83.

[4] W.B. Jeong, et al., Finite element vibration analysis of cylindrical shells conveying fluid with considering acoustic-structure interactions. Jsme International Journal Series C-Mechanical Systems Machine Elements and Manufacturing, Vol.49(2006), p. 488-493. 